\title{
O Sistema Único de Saúde nas dissertações e teses em Educação Física na região Sul do Brasil (2010-2019)
}

\section{The Unified Health System in master's and doctoral theses in Physical Education in the southern region of Brazil (2010-2019)}

\section{El Sistema Único de Salud en tesis de maestría y doctorado en Educación Física en la región Sur de Brasil (2010-2019)}

iD Mathias Roberto Loch

Universidade Estadual de Londrina, Londrina, Paraná, Brasil. e-mail: mathiasuel@hotmail.com

(iD) Joamara de Oliveira Pimentel

Universidade Estadual de Londrina, Londrina, Paraná, Brasil. e-mail: joamarauel@hotmail.com

iD Lean Alécio de Paula Lima Universidade Estadual de Londrina, Londrina, Paraná, Brasil e-mail: lenoxlima@hotmail.com

iD Francine Nesello Melanda Instituto de Saúde Coletiva, Universidade Federal de Mato Grosso, Cuiaba, Mato Grosso, Brasil e-mail: franesello@gmail.com

Resumo: O objetivo foi o de revisar dissertações e teses que apresentavam relação com o Sistema Único de Saúde (SUS) dos Programas de Pós Graduação Stricto Sensu em Educação Física (PPGEF) da região Sul do Brasil, entre 2010 e 2019. A seleção dos trabalhos foi feita por meio da identificação das dissertações e teses publicadas nos sites dos PPGEF, 
seguida da leitura dos títulos e resumos. Foram incluídos 38 (27 dissertações e 11 teses) dos 2163 trabalhos de seis diferentes PPGEF (dois programas não tiveram nenhum trabalho). 23 trabalhos tiveram abordagem quantitativa, 10 qualitativa e cinco eram quali-quanti. Os estudos abrangeram 11 diferentes temáticas. Esses achados reforçam que o SUS ainda é pouco presente nos PPGEF analisados e há grande diversidade de temas investigados.

Palavras-chaves: Sistema Único de Saúde. Educação Física. Saúde Pública. Capacitação Profissional.

Abstract: The objective was to review master's and doctoral theses that were related to the Unified Health System (SUS) of the Stricto Sensu Graduate Programs in Physical Education (PPGEF) in the southern region of Brazil, between 2010 and 2019. The selection of works was done by identifying the dissertations and theses published on the PPGEF websites, followed by reading the titles and abstracts. It included only 38 (27 dissertations and 11 theses), the 2163 work of six different PPGEF (two programs had no work). 23 studies had a quantitative approach, 10 qualitative and five were quali-quanti. The studies covered 11 different themes. These findings reinforce that SUS is still little present in the analyzed PPGEF and there is a great diversity of investigated themes.

Keywords: Unified Health System. Physical Education. Public Health. Professional Training.

Resumém: El objetivo fue revisar las tesis de maestría y doctorado relacionadas con el Sistema Único de Salud (SUS) de los Programas de Posgrado en Educación Física Stricto Sensu (PPGEF) en la región sur de Brasil, entre 2010 y 2019.La selección de trabajos se realizó mediante la identificación de las tesis en los sitios web del PPGEF, seguido de la lectura de títulos y resúmenes. Se incluyeron 38 tesis (27 maestrías y 11 doctorados), de los 2163 trabajos de seis PPGEF diferentes (dos programas no tenían trabajos). 23 estudios tuvieron un enfoque cuantitativo, 10 cualitativos y cinco fueron cuali-cuantitativos. Los estudios abordaron 11 temas diferentes. Estos hallazgos refuerzan que el SUS aún está poco 
presente en los programas analizados y existe una gran diversidad de temas investigados.

Palabras-Clave: Sistema Único de Salud. Educación Física. Salud Pública. Capacitación Profesional.

Submetido em: 07-07-2020

Aceito em: 22-09-2020 
O Sistema Único de Saúde nas dissertações e teses em Educação Física na região Sul do Brasil... Mathias Roberto Loch • Joamara de Oliveira Pimentel • Lean Alécio de Paula Lima • Francine Nesello Melanda

\section{Introdução}

A inserção da Educação Física na Saúde Coletiva tem sido objeto de estudos e reflexões (BAGRICHEVSKY; PALMA; ESTEVÃO, 2003; BAGRICHEVSKY et. al., 2006; PASQUIM, 2010; SANTOS; BENEDETTI, 2012; FALCl; BELISÁRIO, 2013; RODRIGUES et al.,2013; ANDRADE et al., 2014; MARTINEZ; SILVA; SILVA, 2014; SAPORETTI;MIRANDA;BELISARIO, 2016; NOGUEIRA; BOSI, 2017; COSTA, 2019; DAHLKE; VAZ, 2020), bem como as práticas corporais/atividade física (PCAF) têm sido incorporadas em diversas políticas públicas no Brasil (BRASIL, 2014; LOCH et al., 2018).

A falta de uma formação profissional mais específica para atuação no contexto do SUS, em consonância com a realidade social e epidemiológica brasileira, é comumente citada nesses trabalhos, pois ainda prevalece uma formação focada nos aspectos biológicos do processo saúde/doença e centrada no atendimento individualizado para a prescrição de exercícios físicos. Nesse sentido, os Programas de Pós-Graduação Stricto Sensu em Educação Física (PPGEF) têm grande responsabilidade, uma vez que um de seus objetivos é formar futuros docentes e pesquisadores, dos quais muitos atuarão nos cursos de formação inicial em Educação Física. Além do mais, esses programas podem receber alunos que já atuem ou irão atuar no SUS, o que pode ser bastante promissor em termos de se estabelecer uma interlocução entre os PPGEF e o SUS.

Sobre os programas de pós-graduação na área da Educação Física, Manoel e Carvalho (2011) mencionam que os primeiros PPGEF no Brasil foram criados entre o final da década de 1970 e início dos anos 1980. Esses autores destacam que nem todos os programas utilizam a expressão "Educação Física" em seus títulos, e identificaram a existência de 21 programas recomendados pela CAPES em outubro de 2009, sendo que a maior partes deles estava localizada na região Sul e Sudeste do país. No caso específico da região Sul, o primeiro programa foi da Universidade Federal de 
O Sistema Único de Saúde nas dissertações e teses em Educação Física na região Sul do Brasil... Mathias Roberto Loch • Joamara de Oliveira Pimentel • Lean Alécio de Paula Lima • Francine Nesello Melanda

Santa Maria. Esse PPGEF foi também o segundo programa do país na área (o primeiro foi da Universidade de São Paulo), criado em 1979. Porém, posteriormente, o programa foi descredenciado e a UFSM voltou a ter um PPGEF somente, em 2012. Funcionando de maneira ininterrupta, o programa mais antigo da região Sul é o da Universidade Federal do Rio Grande do Sul (UFRGS), que iniciou o mestrado em 1989 e o doutorado em 1999 (QUADROS, AFONSO E RIBEIRO, 2013).

Dado esse contexto, este estudo teve como objetivo revisar dissertações e teses que apresentavam relação com o Sistema Único de Saúde (SUS) dos Programas de Pós Graduação Stricto Sensu em Educação Física (PPGEF) da região Sul do Brasil, entre 2010 e 2019.

\section{Métodos}

Por se tratar de uma revisão de literatura, para a identificação das dissertações e teses em Educação Física, que abordavam o SUS, realizou-se, inicialmente, uma busca junto ao sítio eletrônico da Coordenação de Aperfeiçoamento de Pessoal de Ensino Superior (CAPES), de todos os PPGEF homologados e em funcionamento em $1^{\circ}$ de julho de 2020, localizados na região Sul do Brasil.

Foram identificados oito programas dentro da área Educação Física, sendo três no estado do Rio Grande do Sul: Universidade Federal de Pelotas (UFPEL), Universidade Federal de Santa Maria (UFSM) e Universidade Federal do Rio Grande do Sul (UFRGS); duas em Santa Catarina: Universidade Estadual de Santa Catarina (UDESC) e Universidade Federal de Santa Catarina (UFSC); e três no Paraná: Universidade Federal do Paraná (UFPR), o programa conveniado entre Universidade Estadual de Maringá e Universidade Estadual de Londrina (UEM/UEL) e a Universidade Norte do Paraná (UNOPAR), sendo esta última a única instituição privada e que possui mestrado profissionalizante (nas outras, os cursos são acadêmicos). Vale ressaltar que nem todos os programas têm, em seu 
O Sistema Único de Saúde nas dissertações e teses em Educação Física na região Sul do Brasil... Mathias Roberto Loch • Joamara de Oliveira Pimentel • Lean Alécio de Paula Lima • Francine Nesello Melanda

título, a expressão "Educação Física". Dois programas são denominados "Ciências do Movimento Humano" (UFRGS e UDESC) e um "Exercício Físico na Promoção da Saúde" (UNOPAR). Para facilitar a escrita do texto, optamos por chamar esses programas também de PPGEF, pois consideramos a área e não a nomenclatura dos programas para essa definição.

A fim de selecionar os trabalhos de interesse, inicialmente foram consultados os sítios eletrônicos das universidades e listadas todas as dissertações e teses publicadas entre os anos de 2010 e 2019 disponíveis. Em seguida, a análise dos trabalhos foi realizada em duas etapas: leitura dos títulos e leitura dos resumos. A análise e seleção dos trabalhos foram feitas de forma independente por dois pesquisadores. A cada etapa, caso houvesse divergências, um terceiro autor era solicitado a julgar, e a decisão final foi tomada por consenso ou maioria.

Os dados de interesse dos trabalhos selecionados foram extraídos e registrados em planilha padronizada, que continha as principais informações sobre cada trabalho. $\mathrm{Na}$ análise dos dados, utilizaram-se elementos da estatística descritiva, especialmente análise de frequência (absoluta e relativa). Foi realizada também análise para identificação dos temas abordados em cada trabalho, agrupados pelos autores, nas seguintes categorias: 1) Associação e/ou efeito das PC/AF em indicadores de saúde; 2) Impacto de intervenções no nível de AF; 3) Estudos que buscaram conhecer os serviços e/ou a atuação profissional e/ou o perfil dos profissionais que atuam com as PCAF; 4) Formação inicial (graduação) em Educação Física; 5) Aspectos relativos à adesão e/ou aderência e/ou fatores determinantes para a prática das PCAF; 6) Implementação/ avaliação de projetos de PCAF e/ou análises relativas a esta implementação/avaliação; 7) Indicadores de atividade física e/ou outros indicadores de saúde em trabalhadores do SUS; 8) Caracterização de usuários do SUS; 9) Experiências dos usuários em relação às Práticas Corporais Integrativas; 10) Indicar/desenvolver instrumentos de medidas para utilização no SUS; e 11) Treinamento/capacitação dos trabalhadores do SUS. Alguns trabalhos foram clas- 
O Sistema Único de Saúde nas dissertações e teses em Educação Física na região Sul do Brasil... Mathias Roberto Loch • Joamara de Oliveira Pimentel • Lean Alécio de Paula Lima • Francine Nesello Melanda

sificados em mais de um tema, já que apresentavam mais de um objetivo e estes poderiam estar relacionados a temas diferentes.

Buscando facilitar a escrita e leitura do texto, optamos pela utilização da expressão PCAF, que tem sido utilizada em documentos oficiais. No entanto, ressaltamos que nem todos os autores utilizam essa nomenclatura. Assim, a expressão "atividade física" somente foi utilizada em contextos em que julgamos inadequados o uso de PCAF, como, por exemplo, ao nos referirmos ao nível de atividade física.

\section{Resultados}

No período de 2010 a 2019, foram identificados 2163 trabaIhos (1671 dissertações e 492 teses). Destes, 2121 foram excluídos após a leitura do título por apresentarem objeto de estudo sem alguma relação com o SUS. Após a leitura dos resumos, outros quatro trabalhos foram excluídos, por tratarem mais especificamente do trabalho de outras profissões. Assim, foram selecionados 38 trabalhos para a presente revisão (27 dissertações e 11 teses). Mais detalhes estão apresentados na Figura 1. 
O Sistema Único de Saúde nas dissertações e teses em Educação Física na região Sul do Brasil... Mathias Roberto Loch • Joamara de Oliveira Pimentel • Lean Alécio de Paula Lima • Francine Nesello Melanda

Figura 1 - Fluxograma de seleção das dissertações e teses para revisão da literatura sobre o SUS publicadas entre 2010-2019 nos Programas de PósGraduação em Educação Física da região Sul do Brasil.

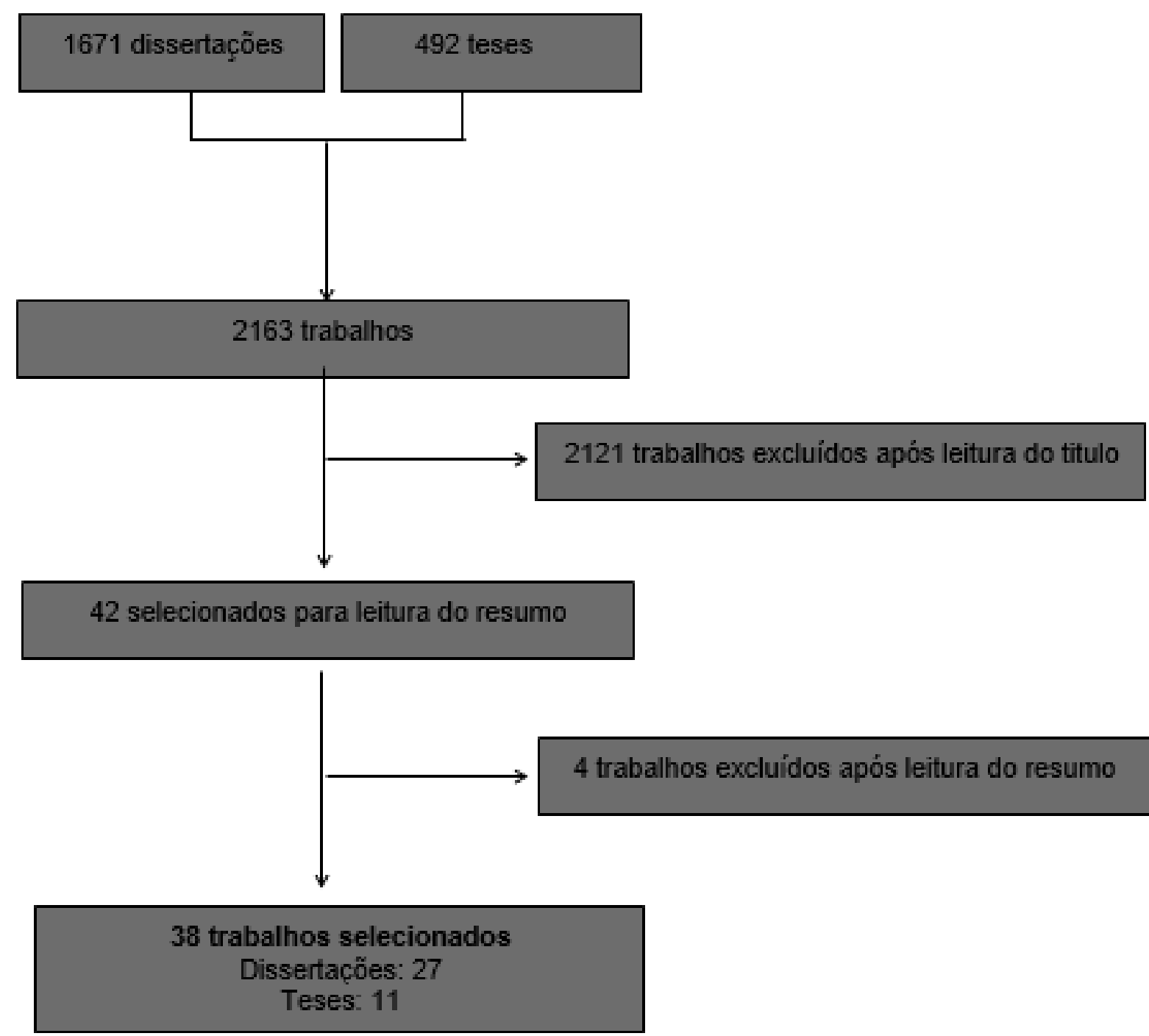

Fonte: Elaboração própria (2020).

A Tabela 1 apresenta dados das Universidades e número de trabalhos (dissertações e teses) encontrados em cada uma (em geral e selecionados para a presente revisão). Observou-se que apenas $1,76 \%(n=38)$ dos trabalhos tinham relação com o SUS. Em números absolutos, o PPGEF da UFSC ( $n=12)$ e da UFPEL $(n=11)$ foram os programas com maior número de trabalhos. Em termos relativos, esses mesmos dois programas apresentaram os maiores percen- 
O Sistema Único de Saúde nas dissertações e teses em Educação Física na região Sul do Brasil... Mathias Roberto Loch • Joamara de Oliveira Pimentel • Lean Alécio de Paula Lima • Francine Nesello Melanda

\begin{abstract}
tuais (4,44\% da UFPEL e 3,4\% da UFSC). Dois (UFSM e UNOPAR) dos oito programas não possuíam nenhum trabalho vinculado ao SUS no período considerado. Porém, há de se considerar que foram os dois programas com menor número de trabalhos defendidos.
\end{abstract}

Tabela 1. Número de dissertações e teses, total e sobre o SUS, segundo Programas de Pós-Graduação em Educação Física da região Sul do Brasil entre 2010-2019.

\begin{tabular}{|c|c|c|c|c|c|c|c|}
\hline UF & $\begin{array}{l}\text { Universidade } \\
\text { (Sigla) }\end{array}$ & $\begin{array}{l}\text { Programa } \\
\text { de Pós- } \\
\text { Graduação }\end{array}$ & $\begin{array}{c}\mathbf{N}^{\circ} \text { de } \\
\text { dissertações }\end{array}$ & $\begin{array}{c}\mathrm{N}^{\circ} \\
\text { de } \\
\text { teses }\end{array}$ & $\begin{array}{l}\text { Total de } \\
\text { trabalhos }\end{array}$ & $\begin{array}{c}N^{\circ} \text { trabalhos } \\
\text { relacionados } \\
\text { ao SUS }\end{array}$ & $\begin{array}{c}\% \text { trabalhos } \\
\text { relacionados } \\
\text { ao SUS }\end{array}$ \\
\hline RS & $\begin{array}{l}\text { Universidade } \\
\text { Federal do Rio } \\
\text { Grande do Sul } \\
\text { (UFRGS) }\end{array}$ & $\begin{array}{l}\text { Ciências do } \\
\text { Movimento } \\
\text { Humano }\end{array}$ & 336 & 146 & 482 & 6 & 1,24 \\
\hline RS & $\begin{array}{l}\text { Universidade } \\
\text { Federal de } \\
\text { Pelotas (UFPEL) }\end{array}$ & $\begin{array}{l}\text { Educação } \\
\text { Física }\end{array}$ & 224 & 24 & 248 & 11 & 4,44 \\
\hline RS & $\begin{array}{l}\text { Universidade } \\
\text { Federal de Santa } \\
\text { Maria (UFSM) }\end{array}$ & $\begin{array}{l}\text { Educação } \\
\text { Física }\end{array}$ & 100 & 0 & 100 & 0 & 0 \\
\hline SC & $\begin{array}{l}\text { Universidade } \\
\text { Federal de Santa } \\
\text { Catarina (UFSC) }\end{array}$ & $\begin{array}{l}\text { Educação } \\
\text { Física }\end{array}$ & 205 & 98 & 303 & 12 & 3,40 \\
\hline SC & $\begin{array}{l}\text { Universidade } \\
\text { do Estado de } \\
\text { Santa Catarina } \\
\text { (UDESC) }\end{array}$ & $\begin{array}{l}\text { Ciências do } \\
\text { Movimento } \\
\text { Humano }\end{array}$ & 282 & 63 & 345 & 3 & 0,87 \\
\hline PR & $\begin{array}{l}\text { Universidade } \\
\text { Federal do } \\
\text { Paraná (UFPR) }\end{array}$ & $\begin{array}{l}\text { Educação } \\
\text { Física }\end{array}$ & 171 & 73 & 244 & 1 & 0,41 \\
\hline PR & $\begin{array}{l}\text { Universidade } \\
\text { Estadual de } \\
\text { Maringá (UEM)/ } \\
\text { Universidade } \\
\text { Estadual de } \\
\text { Londrina (UEL) }\end{array}$ & $\begin{array}{l}\text { Educação } \\
\text { Física }\end{array}$ & 285 & 88 & 373 & 5 & 1,34 \\
\hline PR & $\begin{array}{l}\text { Universidade } \\
\text { Norte do Paraná } \\
\text { (UNOPAR)* }^{*}\end{array}$ & $\begin{array}{l}\text { Exercício } \\
\text { Físico na } \\
\text { Promoção } \\
\text { da Saúde }\end{array}$ & 68 & 0 & 68 & 0 & 0 \\
\hline & TOTAL & & 1671 & 492 & 2163 & 38 & 1,76 \\
\hline
\end{tabular}

* Única universidade particular e o único programa que é profissionalizante. As demais são universidades públicas, com programas de mestrado e doutorado acadêmicos. Fonte: Elaboração própria (2020). 
O Sistema Único de Saúde nas dissertações e teses em Educação Física na região Sul do Brasil... Mathias Roberto Loch • Joamara de Oliveira Pimentel • Lean Alécio de Paula Lima • Francine Nesello Melanda

A figura 2 apresenta a evolução temporal do número de dissertações, teses e o total ao longo do período analisado (2010-2019). Nos quatro primeiros anos (2010 a 2013), o número de trabalhos variou de zero a dois. A partir de 2014, nenhum dos anos teve menos de três trabalhos. Os anos de 2016 e 2017 tiveram o maior número de trabalhos (sete no total, sendo cinco dissertações e duas teses em cada ano), porém, após esse ano, o número de trabalhos tem diminuído, com cinco em 2018 e quatro em 2019.

Figura 2. Números absolutos de trabalhos relacionados SUS em Educação Física do Brasil, segundo ano de publicação (2010-2019).

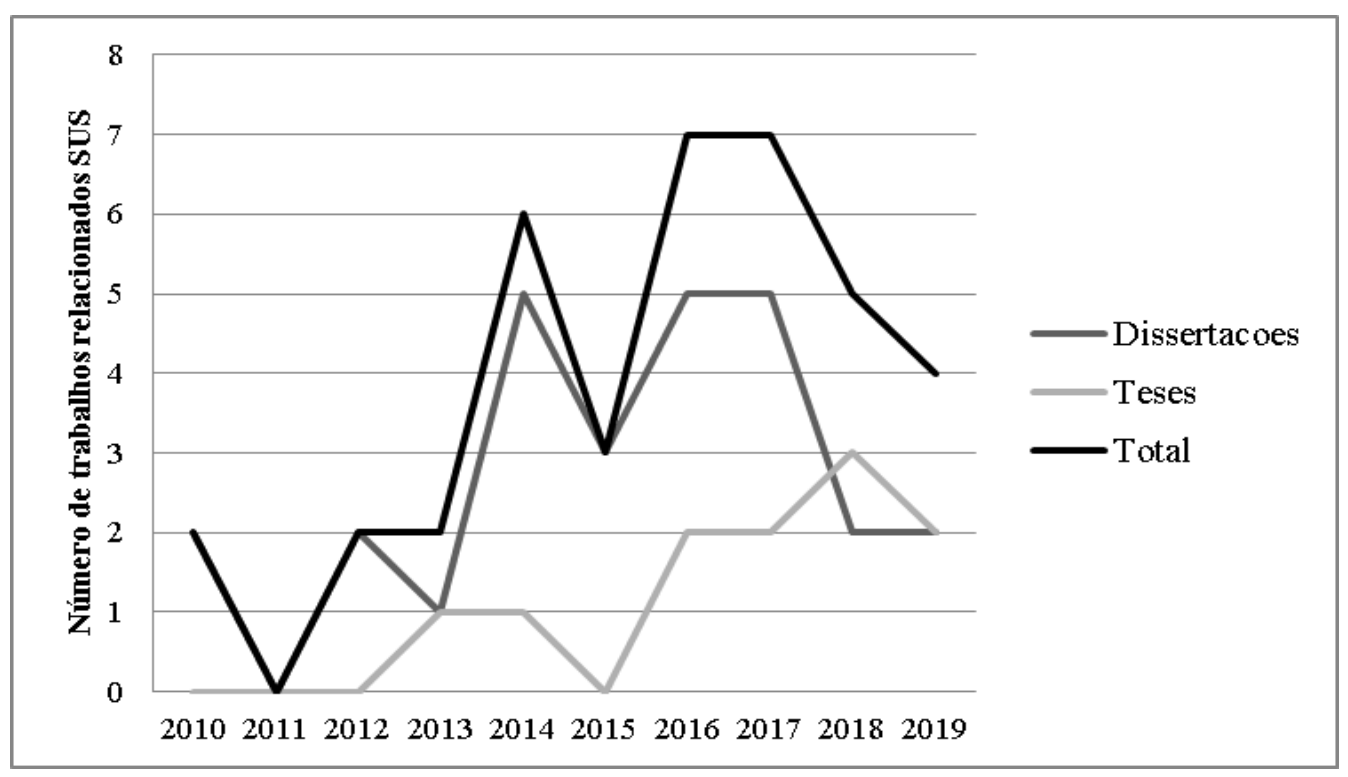

Fonte: Elaboração própria (2020).

O Quadro 1 apresenta os dados das dissertações e teses selecionadas. São apresentadas informações sobre a universidade, o(a) autor(a), ano de defesa, o nível do trabalho (mestrado ou doutorado), a abordagem (quantitativa, qualitativa ou quali-quanti) e o(s) tema(s) de cada trabalho. 
O Sistema Único de Saúde nas dissertações e teses em Educação Física na região Sul do Brasil... Mathias Roberto Loch •Joamara de Oliveira Pimentel • Lean Alécio de Paula Lima • Francine Nesello Melanda

Quadro 1 - Universidade, autor(a), ano, abordagem e tema(s) das dissertações e teses com aproximação com o SUS publicadas entre 2010-2019 nos Programas de Pós-Graduação em Educação Física da região Sul do Brasil.

\begin{tabular}{|c|c|c|c|c|}
\hline Universidade & Autor(a) (ano) & Nível & Abordagem & Tema(s) \\
\hline UFPEL & Jeronimo (2014) & Mestrado & Quantitativa & 7 \\
\hline UFPEL & Silva (2015) & Mestrado & Quantitativa & 2 \\
\hline UFPEL & Hafele (2016) & Mestrado & Quantitativa & 2 \\
\hline UFPEL & Mendes (2017) & Mestrado & Quantitativa & 1 \\
\hline UFPEL & Penny (2017) & Mestrado & Quantitativa & 5 \\
\hline UFPEL & Locatelli (2017) & Mestrado & Quantitativa & 8 \\
\hline UFPEL & Souza (2018) & Mestrado & Quantitativa & 1 \\
\hline UFPEL & Nicoes (2019) & Mestrado & Qualitativa & 6 \\
\hline UFPEL & Correa (2018) & Doutorado & Quantitativa & 1 \\
\hline UFPEL & Seus (2018) & Doutorado & Quantitativa & 3 \\
\hline UFPEL & Galliano (2018) & Doutorado & Quantitativa & 2 \\
\hline UFRGS & Santos (2016) & Mestrado & Qualitativa & 4 \\
\hline UFRGS & Chaise (2015) & Mestrado & Quantitativa & 7 \\
\hline UFRGS & Skowronski (2014) & Mestrado & Qualitativa & 3 \\
\hline UFRGS & Ferreira (2013) & Mestrado & Qualitativa & 3 \\
\hline UFRGS & Bueno (2012) & Mestrado & Qualitativa & 3 \\
\hline UFRGS & Antunes (2019) & Doutorado & Qualitativa & 9 \\
\hline UDESC & Aguiar (2019) & Mestrado & Quantitativa & 5 \\
\hline UDESC & Sandreschi (2016) & Mestrado & Quantitativa & 6 \\
\hline UDESC & Petreça (2017) & Doutorado & Quantitativa & 3,6 \\
\hline UFSC & Soares (2010) & Mestrado & Quantitativa & 8 \\
\hline UFSC & Invernizzi (2010) & Mestrado & Qualitativa & 1 \\
\hline UFSC & Santos (2012) & Mestrado & Quantitativa & 6 \\
\hline UFSC & Scherer (2014) & Mestrado & Quantitativa & 1 \\
\hline UFSC & Borges (2014) & Mestrado & Quali-quanti & 6 \\
\hline UFSC & Tonosaki (2016) & Mestrado & Quali-quanti & 5 \\
\hline UFSC & Silva (2017) & Mestrado & Quali-quanti & 10 \\
\hline UFSC & Gomes (2013) & Doutorado & Quantitativa & 2 \\
\hline UFSC & Fonseca (2014) & Doutorado & Quantitativa & 3 \\
\hline UFSC & Meurer (2016) & Doutorado & Quali-quanti & 2 \\
\hline UFSC & Gerage (2016) & Doutorado & Quantitativa & 1,2 \\
\hline UFSC & José (2017) & Doutorado & Quantitativa & 11 \\
\hline UFPR & Becker (2016) & Mestrado & Quali-quanti & 6 \\
\hline UEM/UEL & Capdeboscq (2014) & Mestrado & Qualitativa & 6 \\
\hline UEM/UEL & Araújo (2015) & Mestrado & Qualitativa & 3 \\
\hline UEM/UEL & Furlan (2016) & Mestrado & Qualitativa & 3 \\
\hline UEM/UEL & leker (2017) & Mestrado & Quantitativa & 7 \\
\hline UEM/UEL & Souto (2018) & Doutorado & Quantitativa & 1 \\
\hline
\end{tabular}

Temas: 1) Associação elou efeito das PC/AF em indicadores de saúde; 2) Impacto de intervenções no nível de AF; 3) Estudos que buscaram conhecer os serviços elou a atuação profissional elou o perfil dos profissionais que atuam com as PCAF; 4) Formação inicial (graduação) em Educação Física; 5) Aspectos relativos à adesão elou aderência elou fatores determinantes para a prática das P(AF; 6) Implementação/avaliação de projetos de PCAF elou análises relativas à esta implementação/avaliação; 7) Indicadores de atividade física elou outros indicadores de saúde em trabalha- 
O Sistema Único de Saúde nas dissertações e teses em Educação Física na região Sul do Brasil... Mathias Roberto Loch • Joamara de Oliveira Pimentel • Lean Alécio de Paula Lima • Francine Nesello Melanda

dores do SUS; 8) Caracterização de usuários do SUS; 9) Experiências dos usuários em relação às Práticas Corporais Integrativas; 10) Indicação de instrumentos de medidas para utilização no SUS; 11) Treinamento/capacitação dos trabalhadores do SUS.

Fonte: Elaboração própria (2020).

Quanto à abordagem dos trabalhos, 23 destes tiveram abordagem quantitativa, 10 qualitativa e cinco eram quali-quanti Em relação aos temas investigados nos trabalhos revisados, observaram-se 11 diferentes temas, sendo os mais frequentes: estudos que buscaram conhecer os serviços e/ou a atuação profissional e/ ou o perfil dos profissionais que atuam com as PCAF $(n=8)$; estudo sobre a associação e/ou efeito das PCAF em indicadores de saúde $(n=7)$, estudos sobre a implementação/avaliação de projetos de PCAF e/ou análises relativas à esta implementação/avaliação $(n=7)$, além de estudos sobre o impacto de intervenções no nível de AF $(n=6)$. Os outros temas identificados foram: estudos sobre aspectos relativos à adesão e/ou aderência e/ou fatores determinantes para a prática das PCAF ( $n=3)$, estudos sobre indicadores de atividade física e/ou outros indicadores de saúde em trabalhadores do SUS ( $n=3)$, estudos caracterizando usuários do SUS ( $n=2)$, além de um trabalho sobre cada um destes temas: estudos com foco na formação inicial (graduação) em Educação Física, experiências dos usuários em relação às Práticas Corporais Integrativas, indicação de instrumentos de medidas para utilização no SUS e treinamento/capacitação dos trabalhadores do SUS.

\section{Discussão}

O presente estudo teve como objetivo principal investigar os trabalhos de mestrado e doutorado dos PPGEF da região Sul do Brasil que apresentavam relação com o SUS no período de 2010 a 2019. Foram verificadas informações de 2163 dissertações ou teses. Com isso, foi constatado que $38(1,76 \%)$ trabalhos apresentavam relação com o SUS. Isso indica que, apesar das novas possibilidades para a atuação do profissional de EF no contexto do SUS, 
O Sistema Único de Saúde nas dissertações e teses em Educação Física na região Sul do Brasil... Mathias Roberto Loch • Joamara de Oliveira Pimentel • Lean Alécio de Paula Lima • Francine Nesello Melanda

essa aproximação ainda é incipiente. Essa revisão também demonstrou grande diversidade quanto aos métodos empregados e nas especificidades do objeto de estudo nos trabalhos analisados.

Quanto ao baixo número de dissertações e teses encontradas, esse achado é coerente com trabalhos que demonstraram que, também no ensino da graduação em Educação Física, a aproximação com o SUS, ou de uma maneira mais ampla, com a Saúde Coletiva, ainda é pequena, parecendo ainda prevalecer fortemente o olhar biomédico (PASQUIM, 2010; FALCl; BELISÁRIO, 2013; ANDRADE et al., 2014; SAPORETTI; MIRANDA; BELISÁRIO, 2016; LOCH, RECH e COSTA, 2020).

É preciso destacar que a Educação Física, muito embora esteja classificada na área da Saúde da CAPES, não se limita à saúde, uma vez que outros objetos/fenômenos/setores fazem parte também do seu escopo, como a educação, o esporte e o lazer. Alguns autores abordam, de maneira bem mais aprofundada, a questão epistemológica da Educação Física e apontam a diversidade da área (BRACHT, 2003; ALMEIDA; VAZ, 2010; ROSA; LETA, 2011). Mesmo com essa ponderação, ressalta-se que foram encontrados apenas 38 em um universo de mais de 2000 trabalhos. Uma proporção baixa para uma área considerada da saúde, se levamos em conta que os PPGEF é que formam os futuros professores e pesquisadores que atuarão como professores no ensino superior. Evidentemente, nem toda dissertação ou tese dos PPGEF, que se relacionam mais à área da saúde, são, necessariamente, vinculados ao SUS. Existem outros "caminhos", como, por exemplo, estudos envolvendo escolas ou escolares, trabalhadores, entre tantos outros. No entanto, vale ressaltar que ainda é muito presente na Educação Física a ideia da saúde como mercadoria, ou mais especificamente, a atividade física como um privilégio restrito àqueles que podem pagar e não como um direito humano básico (LOCH et al., 2018). Por outro lado, há de se ressaltar que nem todo estudo que é realizado no contexto do SUS é "vanguarda" ou esteja isento de críticas. Porém, entendemos que, pelo menos em linhas gerais, os trabalhos mais vinculados ao SUS buscam construir a ideia de 
O Sistema Único de Saúde nas dissertações e teses em Educação Física na região Sul do Brasil... Mathias Roberto Loch • Joamara de Oliveira Pimentel • Lean Alécio de Paula Lima • Francine Nesello Melanda

que o acesso às PCAF e, de maneira mais ampla, à saúde seja um direito de todos.

Na Educação Física, existe grande variedade epistemológica no que se refere aos conceitos e terminologias usadas nas áreas de concentração e linhas de pesquisa. Esse fato, inclusive, pode gerar dificuldades no estabelecimento de suas fronteiras. Porém, o SUS, dadas as suas especificidades, pode ser um espaço importante de "encontro" entre diferentes "modos de olhar". Isso, de algum modo, está evidenciado pela diversidade dos temas dos trabalhos revisados. Essa observação é coerente com o trabalho de Nogueira e Bosi (2017), que apontou distanciamentos e interfaces entre os campos da Educação Física e da Saúde Coletiva. Além disso, observaram tensões que marcam a conformação do campo da Educação Física entre o que as autoras chamam de paradigma biológico e social. Ao mesmo tempo, elas lembram que as reflexões abarcadas pelas Ciências Humanas e Sociais em Educação Física auxiliaram na reorientação do que a Educação Física reconhece como Saúde.

Uma das limitações deste trabalho é ter avaliado somente os PPGEF da região Sul do Brasil. Inicialmente, a intenção era abranger todo o país, entretanto, em função do grande número de trabalhos encontrados, optamos por delimitarmos à região Sul. Além disso, alguns profissionais com formação inicial em Educação Física podem concluir mestrado e doutorado em outras áreas, como em Saúde Coletiva, e realizar pesquisas que tenham relação com o SUS. Porém, é certo que, em termos percentuais, a maior parte dos mestres e doutores (bem como dos mestrandos e doutorandos) brasileiros com formação inicial em Educação Física sejam egressos ou estejam cursando programas específicos da área. Além disso, este trabalho não avaliou artigos e é possível que alguns trabalhos tenham sido publicados em periódicos científicos sem necessariamente serem provenientes de programas de mestrado e doutorado. Contudo, sabe-se que a maior parte da pesquisa no Brasil é realizada nos programas de pós-graduação e muitos dos artigos publicados são baseados em dissertações e teses, ao 
O Sistema Único de Saúde nas dissertações e teses em Educação Física na região Sul do Brasil... Mathias Roberto Loch • Joamara de Oliveira Pimentel • Lean Alécio de Paula Lima • Francine Nesello Melanda

passo que nem toda dissertação ou tese acaba sendo publicada em formato de artigo, o que pode ser um ponto importante deste trabalho. Cabe ressaltar também que essa revisão se baseou nos sites dos PPGEF como fonte dos trabalhos. É possível que nem todas as instituições os mantenham atualizados ou que exista certa morosidade em disponibilizar os trabalhos. Ainda assim, feitas algumas experiências, foi a fonte que julgamos mais apropriada e completa. Este estudo não avaliou a qualidade dos trabalhos, o que inclusive seria bastante difícil dada a grande diversidade de temas e de métodos adotados nos trabalhos revisados. Estudos futuros poderiam focar na análise das dissertações e teses de outras regiões brasileiras, bem como em artigos ou mesmo na produção de trabalhos de iniciação científica e de conclusão de cursos, mesmo que sejam em contextos específicos (uma vez que estes dados não estão disponíveis facilmente em todas as universidades). Vale destacar que, apesar das limitações apontadas, este trabalho investigou um universo grande de dissertações e teses, em um período que pode ser considerado de grande importância para a consolidação da Educação Física enquanto profissão atuante no SUS, além de fornecer elementos importantes para a reflexão sobre as aproximações (ou falta de) dos estudos da pós-graduação brasileira em Educação Física com o SUS.

Os achados deste estudo indicam que, apesar do crescimento do número de estudos envolvendo mais diretamente o SUS ter apresentado uma tendência no período investigado, o tema proposto aqui ainda é pouco presente nos PPGEF analisados. Ademais, observamos uma grande diversidade metodológica e relativa ao objeto de estudo dos trabalhos. Nesse sentido, poderia haver uma maior indução da CAPES e de órgãos de fomento a estudos que sejam mais próximos ao SUS e que os docentes envolvidos nos PPGEF no Brasil considerem a possibilidade de realizarem uma maior aproximação com o SUS. Porém, é importante considerar que, para isso, não basta simplesmente aplicar uma determinada técnica ou intervenção em usuários do SUS da mesma forma como é feito tradicionalmente em outros ambientes (academias 
O Sistema Único de Saúde nas dissertações e teses em Educação Física na região Sul do Brasil... Mathias Roberto Loch • Joamara de Oliveira Pimentel • Lean Alécio de Paula Lima • Francine Nesello Melanda

de ginástica, laboratórios etc.). Faz-se necessário que os pesquisadores se apropriem da especificidade do SUS, principalmente de seus princípios, pois, em alguns casos, a lógica é muito diferente da clínica e privada. Evidentemente, cada caso precisa ser considerado na sua especificidade, mas sugere-se que os PPGEF se aproximem do SUS, buscando compreender as especificidades e complexidades do sistema.

\section{Referências}

AGUIAR, M.S. Implicações das crenças na terapêutica medicamentosa sobre as opções de tratamento pela prática de atividade física em usuários da Atenção Primária em Saúde. 2019. 138f. Dissertação (Mestrado em Ciências do Movimento Humano) - Universidade Estadual de Santa Catarina, Florianópolis (SC), 2019. Disponível em: https://www.udesc.br/cefid/ppgcmh/tesesedissertacoes/dissertacoes4\#2019. Acesso em 30 de julho de 2020. ALMEIDA, F. Q.; VAZ, A. F. Do giro linguístico ao giro ontológico na atividade epistemológica em educação física. Movimento, Porto Alegre, v. 16, n. 3, p. 11-18, 2010.

ANDRADE, D. R.; COSTA, E. F.; GARCIA, L. M. T.; FLORINDO, A. A. Formação do bacharel em educação física frente à situação de saúde no Brasil. In: BENEDETTI, T. R. B.; SILVA, D. A. S.; SILVA, K. S.; NASCIMENTO, J. V. (Org.). A formação do profissional de Educação Física para o setor saúde. Florianópolis: Postmix, p.87-107. 2014.

ANTUNES, P. C. Práticas corporais integrativas: experiências de contracultura na Atenção Básica e emergência de um conceito para o campo da saúde. 2019. 471f. Tese (Doutorado em Ciências do Movimento Humano) - Universidade Federal do Rio Grande do Sul, Porto Alegre (RS), 2019. Disponível em: https://lume.ufrgs. br/handle/10183/203795. Acesso em 30 de julho de 2020. 
O Sistema Único de Saúde nas dissertações e teses em Educação Física na região Sul do Brasil... Mathias Roberto Loch • Joamara de Oliveira Pimentel • Lean Alécio de Paula Lima • Francine Nesello Melanda

ARAUJO, M. S. Os sentidos das representações sociais sobre a intervenção do profissional de educação física na estratégia saúde da família 2015. 140f. Dissertação (Mestrado em Educação Física) - Universidade Estadual de Maringá/ Universidade Estadual de Londrina, Londrina (PR), 2015. Disponível em: https://sucupira.capes.gov.br/sucupira/public/ consultas/coleta/trabalhoConclusao/viewTrabalhoConclusao. jsf?popup=true\&id_trabalho=2506394. Acesso em: 29 out. 2018. BAGRICHEVSKY, M.; PALMA, A.; ESTEVÃO, A. (orgs). A saúde em debate na Educação Física. Blumenau: Edibes, 2003. 191 p. BAGRICHEVSKY, M.; PALMA, A.; ESTEVÃO, A.; DA ROS, M. (orgs). A saúde em debate na Educação Física - volume 2. Blumenau: Nova Letra; 2006. 240 pp.

BECKER, L. A. Tomada de decisão baseada em evidências para prevenção de doenças crônicas não transmissíveis e promoção da atividade física na atenção primária de saúde no estado do Paraná. 2016. 134f. Dissertação (Mestrado em Educação Física) - Universidade Federal do Paraná, Curitiba, 2016. Disponível em: https://acervodigital.ufpr.br/bitstream/handle/1884/42455/R\%20 -\%20D\%20-\%20LEONARDO\%20AUGUSTO\%20BECKER.pdf?sequence=1\&isAllowed=y. Acesso em: 10 jan. 2019.

BORGES, R. A. Programa de promoção de atividade física no SUS: barreiras e facilitadores organizacionais. 2014. $125 \mathrm{f}$. Dissertação (Mestrado em Educação Física) - Universidade Federal de Santa Catarina, Florianópolis, 2014. Disponível em: https://repositorio.ufsc.br/xmlui/bitstream/handle/123456789/129327/329527. pdf?sequence=1\&isAllowed=y. Acesso em: 10 out. 2016.

BRACHT V. Educação física \& ciência: cenas de um casamento (in)feliz. 2. ed. Ijuí: Unijuí; 2003.

BRASIL. Ministério da Saúde. Portaria n. 154, de 24 de janeiro de 2008. Cria os Núcleos de Apoio à Saúde da Família - NASF. Diário Oficial da República Federativa do Brasil, 04 mar 2008; Seção I. 
O Sistema Único de Saúde nas dissertações e teses em Educação Física na região Sul do Brasil... Mathias Roberto Loch • Joamara de Oliveira Pimentel • Lean Alécio de Paula Lima • Francine Nesello Melanda

BRASIL. Ministério da Saúde. Portaria n² 2446, de 11 de novembro de 2014. Redefine a Política Nacional de Promoção da Saúde (PNPS). Diário Oficial da União, 19 nov. 2014. Seção 1, p. 68.

\section{BUENO, A. X. Entre o fazer e o registrar da Educação}

Física no NASF: a relação conflitante entre a Classificação Brasileira de Ocupações e os procedimentos possíveis de registro pelo Profissional de Educação Física. 2012. $106 f$. Dissertação (Mestrado em Ciências do Movimento Humano) Universidade Federal do Rio Grande do Sul, Porto Alegre, 2012. Disponível em: https://www.lume.ufrgs.br/bitstream/handle/10183/67161/000872630. pdf?sequence=1\&isAllowed=y. Acesso em: 10 out. 2016.

\section{CAPDEBOSCQ, M.C. Política públicas de promoção da saúde e} da atividade física: programas e ações realizados no município de Maringá-PR. 2014. 75f. Dissertação (Mestrado em Educação Física) - Universidade Estadual de Maringá/Universidade Estadual de Londrina, Maringá (PR), 2016. Disponível em: http://nou-rau. uem.br/nou-rau/document/?code=vtls000215228. Acesso em 30 de julho de 2020.

CHAISE, F.O. Fatores de riscos à saúde cardiovascular e osteomuscular relacionados aos hábitos, estilo de vida e ao trabalho em profissionais do SAMU 192 Porto Alegre/RS. 2015. 79f. Dissertação (Mestrado em Ciências do Movimento Humano) - Universidade Federal do Rio Grande do Sul, Porto Alegre, 2012. Disponível em: https://lume.ufrgs.br/bitstream/ handle/10183/122649/000972093.pdf?sequence=1. Acesso em 30 de julho de 2020.

CORRÊA, L. Q. Efeito de um programa de exercícios físicos em mulheres diabéticas usuárias de Unidades Básicas de Saúde na cidade de Pelotas-RS: ensaio clínico randomizado. 2018. 232f. Tese (Doutorado em Educação Física) - Universidade Federal de Pelotas, Pelotas, 2018. Disponível em: https://wp.ufpel.edu.br/ ppgef/files/2019/02/Leandro-Quadro-Corr\%C3\%AAa.pdf. Acesso em 30 de julho de 2020. 
O Sistema Único de Saúde nas dissertações e teses em Educação Física na região Sul do Brasil... Mathias Roberto Loch • Joamara de Oliveira Pimentel • Lean Alécio de Paula Lima • Francine Nesello Melanda

COSTA, F. F. Novas diretrizes curriculares para os cursos de graduação em Educação Física: oportunidades de aproximação com o SUS? [editorial] Rev. Bras. Ativ. Fís. Saude, Florianópolis, 2019. DAHLKE, A. P.; VAZ, F. F. Scoping review: práticas corporais na atenção básica em saúde. Pensar a Prática, Goiânia, v.23, 2020. FALCI, D. M.; BELISÁRIO, A.S. A inserção do profissional de Educação Física na atenção primária à saúde e os desafios em sua formação. Interface Comunic. Saúde, Educ., Botucatu, v. 17, n. 47, p. 885-99, 2013. Disponível em: http://dx.doi.org/10.1590/ S1414-32832013005000027. Acesso em: 5 dez. 2018.

FERREIRA, L. A. S. 0 trabalho da educação física na composição de equipes da saúde mental especializada em álcool e outras drogas 2013. 108f. Dissertação (Mestrado em Ciências do Movimento Humano) - Universidade Federal do Rio Grande do Sul, Porto Alegre, 2013. Disponível em: https://lume.ufrgs.br/bitstream/handle/10183/99039/000930194.pdf?sequence=1. Acesso em 30 de julho de 2020.

FONSECA AS. Promoção da atividade física para gestantes na atenção básica à saúde da região metropolitana de Recife: projeto Sus+ativo. 2014, 125f. Tese (Doutorado em Educação Física) - Universidade Federal de Santa Catarina, Florianópolis, 2014. Disponível em: http://tede.ufsc.br/teses/PGEF0446-T.pdf. Acesso em 30 de julho de 2020

FURLAN, A. A. 0 trabalho do núcleo de apoio a saúde da família (nasf) e da educação física na atenção básica: em busca de sentido. 2016. 114f. Dissertação (Mestrado em Educação Física) - Universidade Estadual de Maringá/Universidade Estadual de Londrina, Maringá (PR), 2016. Disponível em: https://sucupira.capes.gov.br/sucupira/public/consultas/coleta/trabalhoConclusao/ viewTrabalhoConclusao.jsf?popup=true\&id_trabalho $=3611478$. Acesso em: 29 out. 2018.

GALLIANO, L. M. Estudo de saúde dona francisca: efeitos de uma intervenção vinculada ao sus sobre desfechos em saúde. 2018. 200f. Tese (Doutorado em Educação Física) - Universidade 
O Sistema Único de Saúde nas dissertações e teses em Educação Física na região Sul do Brasil... Mathias Roberto Loch • Joamara de Oliveira Pimentel • Lean Alécio de Paula Lima • Francine Nesello Melanda

Federal de Pelotas, Pelotas, 2018. Disponível em: https:// sucupira.capes.gov.br/sucupira/public/consultas/coleta/trabaIhoConclusao/viewTrabalhoConclusao.jsf?popup=true\&id_trabaIho=7643270. Acesso em 30 de julho de 2020.

GARCIA, L.; BÖHM, A.; BACIL, E.; CRUZ, M.; SANTO, R. E. A inserção da subárea de Atividade Física e Saúde nos programas de pós-graduação em Educação Física no Brasil. Rev. Bras. Ativ. Fís. Saude [online], Florianópolis, v. 19, n. 2, p. 215-222, 2014. Disponível em: http://dx.doi.org/10.12820/rbafs.v.19n2p215 . Acesso em: 10 abr. 2019.

GERAGE, A. M. Programa Vida Ativa Melhorando a Saúde: efetividade sobre variáveis comportamentais e de risco cardiovascular em pacientes hipertensos. 2016. 156f. Tese (Doutorado em Educação Física) - Universidade Federal de Santa Catarina, Florianópolis, 2016. Disponível em: http://tede.ufsc.br/teses/ PGEF0430-T.pdf. Acesso em 30 de julho de 2020.

GOMES, M. A. A educação física do Sistema Único de Saúde (SUS): efetividade de uma intervenção de base comunitária para promoção da atividade e saúde. 2013. 191f. Tese (Doutorado em Educação Física) - Universidade Federal de Santa Catarina, Florianópolis, 2013. Disponível em: https://repositorio.ufsc.br/ handle/123456789/107056. Acesso em: 10 out. 2016.

HÄFELE V. Aconselhamento para a prática de atividade física e estágios de mudança de comportamento dos usuários das Unidades Básicas de Saúde da cidade de Pelotas/RS. 2016. 118f. Dissertação (Mestrado em Educação Física) - Universidade Federal de Pelotas, Pelotas (RS), 2016. Disponível em: http:// repositorio.ufpel.edu.br:8080/bitstream/prefix/3760/1/Vitor\%20 Hafele.pdf. Acesso em: 4 dez. 2018.

IEKER, A.S.D. Nível de atividade física, perfil de saúde e fatores de risco para doenças crônicas em servidores de um hospital escola do sul do Brasil. 2017. 56f. Dissertação (Mestrado em Educação Física) - Universidade Estadual de Maringá/Universidade Estadual de Londrina, Maringá (PR), 2017. 
O Sistema Único de Saúde nas dissertações e teses em Educação Física na região Sul do Brasil... Mathias Roberto Loch • Joamara de Oliveira Pimentel • Lean Alécio de Paula Lima • Francine Nesello Melanda

Disponível em: http://nou-rau.uem.br/nou-rau/document/?code=vtls000226128. Acesso em 30 de julho de 2020.

JERONIMO, J.S. Atividade física e fatores associados em trabaIhadores de Centros de atenção psicossocial da região sul do Brasil: tendências temporais 2006 - 2011. 2014. 120f. Dissertação (Mestrado em Educação Física) - Universidade Federal de Pelotas, Pelotas, 2014. Disponível em: https://wp.ufpel.edu.br/ppgef/ files/2016/03/Jeferson-Santos-Jer\%C3\%B4nimo.pdf. Acesso em 30 de julho de 2020.

JOSÉ, H.P.M. Avaliação do treinamento de multiplicadores do Programa Vida Ativa Melhorando a Saúde - VAMOS. 2017. 308f. Tese (Doutorado em Educação Física) - Universidade Federal de Santa Catarina, Florianópolis, 2017. Disponível em: http://tede.ufsc.br/teses/PGEF0470-T.pdf. Acesso em 30 de julho de 2020.

LOCATELLI, L. Uso de drogas e aspectos de saúde em pacientes de um Centro de Atenção Psicossocial em Álcool e outras Drogas III de Pelotas/RS 2016. 100f. Dissertação (Mestrado em Educação Física) - Universidade Federal de Pelotas, Pelotas, 2017. Disponível em: https://wp.ufpel.edu.br/ppgef/files/2018/03/ Disserta\%C3\%A7\%C3\%A3o-Liliane-Locatelli.pdf. Acesso em 30 de julho de 2020.

LOCH, M.R; KNUTH, A. G.; SILVA, I. C. M; GUERRA, P. H. As práticas corporais/atividade física nos 30 anos do Sistema Único de Saúde. Ciên.

Saúde Colet., Rio de Janeiro, v. 23, n. 10, p. 3469-3469.

LOCH, M.R.; RECH, C.R.; COSTA, F.F. A urgência da Saúde Coletiva na formação em Educação Física: lições com o COVID-19. Ciên. Saúde Colet., Rio de Janeiro, v. 25, n. 9, p. 3511-3516.

MANOEL, E. J.; CARVALHO, Y. M. Pós-Graduação na Educação Física Brasileira: a atração (fatal) para a biodinamica. Educação e Pesquisa (USP. Impresso), São Paulo, v. 37, p. 389-406, 2011. MENDES, G. F. F. Independência funcional e nível de atividade física de lazer e deslocamento em idosos usuários das Unidades Básicas de Saúde da cidade de Pelotas/RS. 2017. 
O Sistema Único de Saúde nas dissertações e teses em Educação Física na região Sul do Brasil... Mathias Roberto Loch • Joamara de Oliveira Pimentel • Lean Alécio de Paula Lima • Francine Nesello Melanda

132f. Dissertação (Mestrado em Educação Física) - Universidade Federal de Pelotas, Pelotas (RS), 2017. Disponível em: https:// wp.ufpel.edu.br/ppgef/files/2018/03/Disserta\%C3\%A7\%C3\%A3oGraciele-F.-Mendes.pdf. Acesso em: 4 dez. 2018.

MEURER, S. T. Avaliação da estratégia de promoção de atividade física e alimentação saudável - vamos - no programa academia da saúde de Belo Horizonte, Minas Gerais. 2016. $184 f$. Tese (Doutorado em Educação Física) - Universidade Federal de Santa Catarina, Florianópolis, 2016. Disponível em: https:// repositorio.ufsc.br/bitstream/handle/123456789/174876/344712. pdf?sequence=1\&isAllowed=y. Acesso em: 11 jan. 2019.

NICOES, C.R. Da Lagoa dos Patos aos Campos Neutrais: a implementação do Núcleo de Apoio à Saúde da Família em dois municípios do extremo Sul do Brasil. 2019. 75f. Dissertação (Mestrado em Educação Física) - Universidade Federal de Pelotas, Pelotas, 2019. Disponível em: https://sucupira.capes.gov.br/ sucupira/public/consultas/coleta/trabalhoConclusao/viewTrabaIhoConclusao.jsf?popup=true\&id_trabalho $=8121473$. Acesso em 30 de julho de 2020.

NOGUEIRA, J. A. D.; BOSI, M. L. M. Saúde Coletiva e Educação Física: distanciamentos e interfaces. Ciên. Saúde Colet., Rio de Janeiro, v. 22, n. 6, p. 1913-1922, 2017.

INVERNIZZI, L. Educação física na classe hospitalar do Hospital Infantil Joana de Gusmão: delineando uma proposta de ensino para os anos iniciais. 2010. 185f. Dissertação (Mestrado em Educação Física) - Universidade Federal de Santa Catarina, Florianópolis, 2010. Disponível em: http://www.tede.ufsc.br/teses/ PGEF0250-D.pdf. Acesso em 30 de julho de 2020.

PASQUIM, H. M. A saúde coletiva nos cursos de graduação em Educação Física. Saúde e Soc., Florianópolis, v. 19, n. 1, p. 193200, 2010.

PENNY, J. C. Auto eficácia Para Prática de Atividade Física em Mulheres Diabéticas Usuárias de Unidade Básica de Saúde na Cidade de Pelotas - RS: Ensaio Clínico Randomizado. 
O Sistema Único de Saúde nas dissertações e teses em Educação Física na região Sul do Brasil... Mathias Roberto Loch • Joamara de Oliveira Pimentel • Lean Alécio de Paula Lima • Francine Nesello Melanda

2017. 145 f. Dissertação de Mestrado (Mestrado em Educação Física), Universidade Federal de Pelotas, Pelotas (RS), 2017. Disponível em: https://wp.ufpel.edu.br/ppgef/files/2018/03/ Disserta\%C3\%A7\%C3\%A3o-Joubert-Caldeira-Penny.pdf. Acesso em: 4 dez. 2018.

PETREÇA, D. R. Avaliação de intervenções em atividade física para idosos e outras populações na saúde pública de Santa Catarina 2017. 215f. Tese (Doutorado em Ciências do Movimento Humano) - Universidade do Estado de Santa Catarina, Florianópolis, 2017. Disponível em: https://sucupira.capes.gov.br/ sucupira/public/consultas/coleta/trabalhoConclusao/viewTrabaIhoConclusao.jsf?popup=true\&id_trabalho=5070183. Acesso em: 10 dez. 2018.

QUADROS, H. M.; AFONSO, M. R.; RIBEIRO, J. A. B. O cenário da pós-graduação em Educação Física: Contextos e possibilidades na região sul do Brasil. Revista Brasileira de Atividade Física \& Saúde, Florianópolis, v. 18, p. 576-584, 2013.

RODRIGUES, J. D. Atuação do profissional de educação física nos núcleos de apoio à saúde da família na região metropolitana de João Pessoa-PB. 2012. 74f. Dissertação (Mestrado em Educação Física) - Universidade de Pernambuco/Universidade Federal da Paraíba, João Pessoa (PB), 2012. Disponível em: http:// w2.files.scire.net.br/atrio/upe-papgef_upl//THESIS/83/jos_damio_rodrigues.pdf. Acesso em: 28 set. 2016.

RODRIGUES, J.D.; FERREIRA, D. K. S.; SILVA, P. A.; CAMINHA, I. O.; FARIAS JUNIOR, J. C. Inserção e atuação do profissional de educação física na atenção básica à saúde: revisão sistemática. Rev. Bras. Ativ. Fís. Saúde, Florianópolis, v. 18, n. 1, p. 5-15. 2013. ROSA, S.; LETA, J. Tendências atuais da pesquisa brasileira em Educação Física Parte 2: a heterogeneidade epistemológica nos programas de pós-graduação. Rev. Bras. Educ. Fís. Esporte [online], São Paulo, v. 25, n. 1, p. 7-18, 2011. Disponível em: http:// dx.doi.org/10.1590/S1807-55092011000100002 . Acesso em: 21 jan. 2019. 
O Sistema Único de Saúde nas dissertações e teses em Educação Física na região Sul do Brasil... Mathias Roberto Loch • Joamara de Oliveira Pimentel • Lean Alécio de Paula Lima • Francine Nesello Melanda

SANDRESCHI, P. F. Secretários municipais de saúde de Santa Catarina: perfil e visão sobre as barreiras para o desenvolvimento de programas de atividade física para idosos. 2016. $123 \mathrm{f}$. Dissertação (Mestrado em Ciências do Movimento Humano) Universidade do Estado de Santa Catarina, Florianópolis, 2016. Disponível em: https://sucupira.capes.gov.br/sucupira/public/ consultas/coleta/trabalhoConclusao/viewTrabalhoConclusao. jsf?popup=true\&id_trabalho=3938396. Acesso em: 10 dez. 2018.

\section{SANTOS, D.F. Educação Física no Hospital Psiquiátrico São}

Pedro: uma experiência de familiarização e estranhamento nas entrelinhas dos relatos de estágio. 2016. 119f. (Mestrado em Ciências do Movimento Humano) - Universidade Federal do Rio Grande do Sul, Porto Alegre, 2016. Disponível em: https://lume. ufrgs.br/handle/10183/151419. Acesso em 30 de julho de 2020.

\section{SANTOS, S. F. S. Núcleo de Apoio à Saúde da Família no}

\section{Brasil e a atuação do profissional de educação física.}

2012. 185f. Dissertação (Mestrado em Educação Física) Universidade Federal de Santa Catarina, Florianópolis, 2012. Disponível em:https://repositorio.ufsc.br/bitstream/handle/123456789/99337/305531. pdf?sequence=1\&isAllowed=y. Acesso em: 10 out. 2016.

SANTOS, S. F. S.; BENEDETTI, T.R.B. Cenário de implantação do Núcleo de Apoio a Saúde da Família e a inserção do profissional de Educação Física. Rev. Bras. Ativ. Fís. Saúde, Florianópolis, v. 17, n. 3, p. 188-194, 2012. Disponível em: http://dx.doi. org/10.12820/rbafs.v.17n3p188-194. Acesso em: 5 dez. 2018. SAPORETTI, G. M.; MIRANDA, P. S. C.; BELISARIO, A. S. O profissional de Educação Física e a promoção da saúde em Núcleos de Apoio à Saúde da Família. Trab. educ. saúde.; v. 14, n. 2, p. 523-543, 2016. Disponível em: http://dx.doi.org/10.1590/ 1981-7746-sip00113. Acesso em: 5 dez. 2018.

SCHERER, F.C. Programas de promoção da saúde: nível de atividade física e qualidade de vida de idosos nos Centros de Saúde de Florianópolis. 2014. 193f. Dissertação (Mestrado em Educação 
O Sistema Único de Saúde nas dissertações e teses em Educação Física na região Sul do Brasil... Mathias Roberto Loch • Joamara de Oliveira Pimentel • Lean Alécio de Paula Lima • Francine Nesello Melanda

Física) - Universidade Federal de Santa Catarina, Florianópolis, 2014. Disponível em: http://tede.ufsc.br/teses/PGEF0376-D.pdf.. Acesso em 30 de julho de 2020.

SEUS, T.L.C. Núcleo de Apoio à Saúde da Família e o Profissional de Educação Física. 2018. 148f. Tese (Doutorado em Educação Física) - Universidade Federal de Pelotas, Pelotas, 2018. Disponível em: https://wp.ufpel.edu.br/ppgef/files/2019/02/ Thamires-Lorenzet-Cunha-Seus.pdf. Acesso em 30 de julho de 2020.

SILVA, W. M. Impacto de intervenção com atividade física em população de área de cobertura de Unidade Básica de Saúde. 2015. 114f. Dissertação (Mestrado em Educação Física) - Universidade Federal de Pelotas, Pelotas (RS), 2015. Disponível em: https://sucupira.capes.gov.br/sucupira/public/consultas/ coleta/trabalhoConclusao/viewTrabalhoConclusao.jsf?popup=true\&id_trabalho=3076242. Acesso em: 4 dez. 2018.

SILVA, M.C. Instrumentos para medida de atividade física, alimentação e antropometria no Programa Vida Ativa Melhorando a Saúde - VAMOS: opiniões dos especialistas e multiplicadores. 2017. 149f. Dissertação (Mestrado em Educação Física) - Universidade Federal de Santa Catarina, Florianópolis, 2017. Disponível em: http://tede.ufsc.br/teses/PGEF0482-D.pdf. Acesso em 30 de julho de 2020.

SOARES, A. Perfil dos indivíduos com obesidade mórbida submetidos à cirurgia bariátrica pelo Sistema Único de Saúde no Hospital Universitário da Universidade Federal de Santa Catarina. 2010. 109f. Dissertação (Mestrado em Educação Física) - Universidade Federal de Santa Catarina, Florianópolis, 2010. Disponível em: http://www.tede.ufsc.br/teses/PGEF0252-D.pdf. Acesso em 30 de julho de 2020.

SOUTO, E. C. Prática de atividade física para indivíduos com lesão medular atendidos no Sistema Único de Saúde de João Pessoa /PB: fatores intervenientes e influência na aptidão física e saúde. 2018. 113f. Tese (Doutorado em Educação Física) 
O Sistema Único de Saúde nas dissertações e teses em Educação Física na região Sul do Brasil... Mathias Roberto Loch • Joamara de Oliveira Pimentel • Lean Alécio de Paula Lima • Francine Nesello Melanda

- Universidade Estadual de Maringá/Universidade Estadual de Londrina, Maringá (PR), 2018. Disponível em: http://www.bibliotecadigital.uel.br/document/?code=vtls000224106. Acesso em 30 de julho de 2020.

SOUZA, D. F. S. Prevalência de dor crônica, nível de atividade física e fatores associados em usuários das unidades básicas de saúde do município de Pelotas/RS. 2018. 122f. Dissertação (Mestrado em Educação Física) - Universidade Federal de Pelotas, Pelotas, 2018. Disponível em: https://wp.ufpel.edu.br/ppgef/ files/2019/02/Daniele-Fernandes-da-S.-de-Souza.pdf. Acesso em 30 de julho de 2020

SKOWRONSKI, M. Educação física e saúde: a mobilização de saberes para atuação nos polos do Programa Academia da Saúde no Brasil. 2014. 130f. Dissertação (Mestrado em Ciências do Movimento Humano) - Universidade Federal do Rio Grande do Sul, Porto Alegre, 2014. Disponível em: https://www.lume.ufrgs. br/bitstream/handle/10183/109123/000949996.pdf?sequence=1\&isAllowed=y. Acesso em: 10 out. 2016.

TONOSAKI, L.M.D. Programa Vida Ativa Melhorando a Saúde (VAMOS): percepção dos participantes quanto à saúde, barreiras e facilitadores. 2016. 100f. Dissertação (Mestrado em Educação Física) - Universidade Federal de Santa Catarina, Florianópolis, 2016. Disponível em: http://tede.ufsc.br/teses/PGEF0437-D.pdf. Acesso em 30 de julho de 2020.

\section{Publisher}

Universidade Federal de Goiás. Faculdade de Educação Física e Dança. Publicação no Portal de Periódicos UFG. As ideias expressadas neste artigo são de responsabilidade de seus autores, não representando, necessariamente, a opinião dos editores ou da universidade. 